SEARCH FOR QGP SIGNALS AT AGS WITH A TPC SPECTROMETER, AND COMPARISON OF OUR EVENT GENERATOR PREDICTIONS FOR PLASMA MODEL AND CASCADE INTERACTIONS ${ }^{\dagger}$

\section{S.J. Lindenbaum}

Brookhaven National Laboratory and City College of New York

K.J. Foley, S.E. Eiseman, A. Etkin, R.W. Hackenburg,

R.S.Longacre, W.A. Love, T.W. Morris, E.D. Platner, A.C. Saulys

Brookhaven National Laboratory, Upton, New York 11973

C. Chan, M.A. Kramer

City College of New York, New York, New York 10031

T.J. Hallman, L. Madansky

Johns Hopkins University, Baltimore, Maryland 21218

B.E. Bonner, J.A. Buchanan, J.M. Clement, M.D. Corcoran, J.W. Kruk, H.E. Miettinen, G.S. Mutchler, F. Nessi-Tedaldi, M. Nessi, G.C. Phillips, J.B. Roberts

Rice University, Houston, Texas 77251

\section{ABSTRACT}

We have developed and successfully tested a TPC Magnetic Spectrometer to search for QGP signals produced by ion beams at AGS. He also developed a cascade and plasma event generator the predictions of which are used to illustrate how our technique can detect possible plasma signals.

\section{INTRODUCTION}

In this talk we presented the following topics under investigation by the BNL/CCNY/Johns Hopkins/Rice Collaboration: 1) Quark Gluon Plasma and ordinary cascade event simulation in ion-nucleus collisions; 2) A TPC Tracking Magnetic Spectrometer system for plasma signal detection; 3) Plasma signals detectable at AGS.

In order to simulate what non-plasma events would do we modified ISAJET to conserve baryon number, strangeness, energy and momentum. We then improved the remainder of HIJET to include cascading of slow produced particles inside the nucleus leading to $N-N, \pi-N$ and $K-N$ scattering. Then we interfaced a version of the fragment program

$\dagger$ This research was supported by the U.S. Department of Energy under Contract Nos. DE-AS05-81ER40032, DE-AC02-76ER03274, DE-ACO2$76 C H 00016, D E-A C 02-83 E R 40107$ and the City University of New York PSC-BHE Research Award Program. 


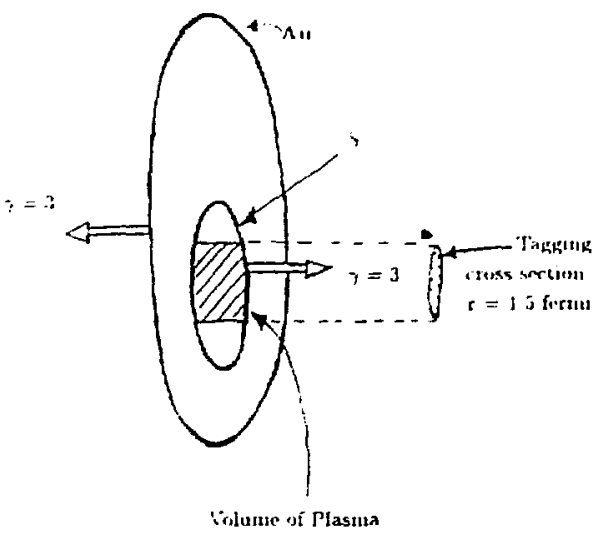

Tugged particles doenraun: the chesucal potentual

Figure la: Tagged Plasma Volume
FAIHI with HIJET. This gave us a more realistic cascade (non-plasma) generator for ordinary events.

\section{Central ordinary cascade} events led to essentially complete stopping (in the nucleon-nucleon cms) of incident sulphur ions by a gold target. This is consistent with experimental results ${ }^{1}$ for AGS energies. As an illustration of what our technique can be expected to do we added a simple plasma model. We assumed that in some of the $15 \mathrm{GeV} / \mathrm{C} \times \mathrm{A}$ sulphur on $A u$ events the nucleon-nucleon central collisions in a tagged region of radius 1.5 fermi were stopped in a compressed volume in the nucleon-nucleon $\mathrm{cm}$ system corresponding to the $r=3$ (Fig. $2 \mathrm{a}$ ). This determined the energy and chemical potential. These tagged nucleons made a spherical plasma bubble of the Van Hove type of critical temperature $170 \mathrm{MeV}$, with chemical potential and volume which conserves baryon number and energy.

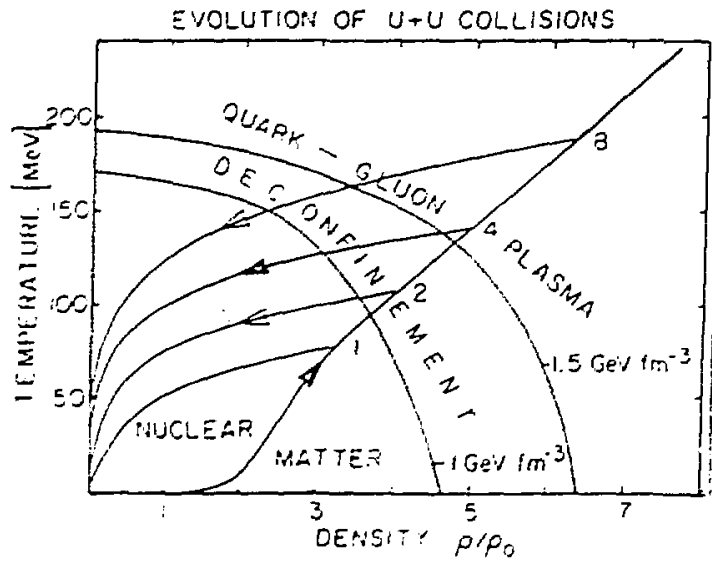

Figure 1b: T vs. $\rho$ (Stocker, Nuc T. Phys. A418, 587C (1984)
If one looks at an example of the canonical temperature vs. pressure diagram (Fig. lb) in conjunction with the dynamics (Fig. 1b) it appears that approximately complete stopping at AGS energies could have a reasonable chance of producing plasma in some of the central events at AGS by providing a combination of high enough baryon density and temperature. The plasma breakup was based on the work. of Koch, Muller and Rafelski. ${ }^{2}$ In their model the particle production probabilities depends on the critical temperature and the gluon fragmentation function. 
We conserve all relevant quantum numbers, energy and momentum. The plasma bubble typically contains $-30 \mathrm{GeV}$ (i.e. $<20 \%$ of the available energy) and spreads over $=1$ unit of rapidity. A given chemical potential $\mu$ and critical temperature $T_{C}$ for the plasma bubble determines $N_{q}, N_{\vec{q}}, N_{S}, N_{s}, N_{d}$ where $N$ stands for the number. The subscripts $q, q, s, s$ and $g$ stand for $(u, d)$ quarks, $(\bar{u}, \bar{d})$ quarks, strange quarks, anti-strange quarks and gluons respectively. The effective number of quarks when they hadronize are: $\tilde{N}_{q}=N_{q}+f_{q} N_{g} ; \quad \tilde{N}_{q}=N_{q}+f_{q} N_{g} ; \tilde{N}_{s}=N_{s}+f_{s} N_{g} ; \tilde{N}_{s}=\tilde{N}_{s}$. where $f_{q}=$ gluon fragmentation function for $u$ and $d$. $f_{s}=$ gluon fragmentation functions for $s$. The average number of particles produced each with a Boltzman distribution corresponding to the critical temperature is: $N_{\pi}=\alpha \tilde{N}_{q} \tilde{N}_{\bar{q}}, N_{K}=\alpha \tilde{N}_{q} \tilde{N}_{\bar{S}}, N_{R}=\alpha \tilde{N}_{q}-\tilde{N}_{S}, N_{\phi}=$ $\alpha \tilde{N}_{S} N_{S}, N_{n}=1 / 3 ! \beta \bar{N}_{q}{ }^{3}, N_{n}=1 / 3 ! \beta \tilde{N}_{\bar{q}}^{-3}, N_{\Lambda}=1 / 2 ! B \tilde{N}_{q} 2 \tilde{N}_{S}, N_{\bar{\Lambda}}=$ $1 / 2 ! B \tilde{N}_{\bar{q}}{ }^{2} \tilde{N}_{\tilde{S}}, N_{\Xi}=1 / 2 ! \beta \tilde{N}_{q} \tilde{N}_{S}{ }^{2}, N \equiv=1 / 2 ! B \tilde{N}_{\bar{Q}} \tilde{N}_{\bar{S}}^{2}, N_{\Omega}=1 / 3 ! B \tilde{N}_{S}{ }^{3}$, $N_{\bar{\Omega}}=1 / 3 ! \tilde{N}_{s}^{-3}$. The plasma production and breakup models we have used may be far from correct. However that may also be true of all others proposed to date.

\section{REQUIREMENTS ON THE DETECTOR SYSTEM}

From the foregoing investigation it is clear that a tracking detector system capable of working in this high particle density environment with sufficient space, angular and momentum resolution and a means of identifying $\bar{p}$ is essential. A TPC is a three dimensional point detector and thus can track in much higher multiplicity enviroments than projective geometry detectors. We have built a specially designed TPC to maximize two-track separability and also minimize costs. As a result $d E / d x$ information for the TPC is not available. However it is supplemented with a time-of-flight hodoscope (TOF) which will identify $*$ half of the $\bar{p}$ particles of interest. Secondly negative particles are expected to be $>90 \%$ pions so that pion identification can be made to a good approximation. Figure 2 shows the experimental arrangement for the first phase of AGS E-810. The TOF $\bar{p}$ identifier is $50^{\prime}$ downstream of target and is not shown in Fig. 2 .

Four modules comprise the TPC system. They are placed along the beam with the $S$ (or $S i$ ) and 0 ion beams passing through the TPC to provide large solid angle coverage. From ion beam tests we conclude that this will be satisfactory for these runs. However it should be noted that one can deaden the beam area if necessary. When the booster becomes available at AGS to accelerate $\mathrm{Au}$ ions; this will likely become necessary. The anode readout wires are 20 s gold-plated tungsten $1 \mathrm{~cm}$ long rows parallel to the beam direction. There are 10 wires to the inch between cathode structures. A gate which opens only when events of interest occur is included for operation at high ion beam rates $\left(-1 / 2 \quad 10^{4} /\right.$ pulse). 
A TPC module was tested in > $25 \mathrm{~K}$ Si ions per pulse beam without magnetic field. The hits can clearly be associated with several tracks consistent with beam particles dispersed in $y$ because of late arrival. When we reduced the anode structure gain to correspond to detecting minimum ionizing particles the silicon ions were detected with high efficiency $>90 \%$. The next tests were in an $18 \mathrm{GeV} / \mathrm{c}$ proton beam. Figure 3a shows accidental tracks from several million per pulse incident $18 \mathrm{GeV} / \mathrm{c}$ protons. Figure 3b shows detected events from a target. Our pattern recognition program led to fits with efficiencies of $>90 \%$. Figure $3 c$ shows the TPC module inside the MPS magnet with a $5 \mathrm{KG}$ field. A $3 \mathrm{GeV} \pi^{-}$beam is incident and low moment um tracks from a target are clearly reconstructed with high efficiency ( $>90 \%$ ). The resolutions in $x$ (transverse to the beam and magnetic field) and $y$ (along the drift and magnetic field direction but perpendicular to the beam) were both measured to be $<1 \mathrm{~mm}$. The two track separability was measured to be $\approx 1-3 \mathrm{~mm}$. The gas is a stable high gain, low difusion mixture of Argon, Isobutane and Methylal which is a slightly modified mixture of the standard MPS II drift chamber gas. Minimum ionizing particles gave clear well-shaped pulses averaging 8 mamps with peaks of $>20$ uamps. Setting a threshold of 2-3 yamps ensured clean pulse operation with very high efficiency. The readout electronics was vintage 86 LeCroy hybrids we designed for TPC readout. An improved version of prototypes has in tests shown that even lower thresholds (i.e. a fraction of a microamp) will be useable with the new readout system.

Thin targets will be usted to minimize secondary interactions. The triggering system includes beam halo counters in veto, counters to detect interactions in the target and a $\left[Z^{2}\right.$ counter to select candidates for events varying from central to peripheral depending on the setting (see Fig. 2). We prefer minimal triggering in the early stages since according to our Monte Carlo's and plasma model one can get surprises if one íriggers tightly. For example our plasma model calculations predict that plasma events have a smaller multiplicity distribution than cascade events at AGS energies. The pattern recognition program previously developed by BNL/CCNY was described in Ref. 3. It has been modified somewhat. A local pattern recognition forms a single hit from adjacent and nearby readout wires. A subroutine corrects $\vec{E} \times \vec{B}$ effects which varies from less than $2 \mathrm{~mm}$ to a fraction of a $\mathrm{mm}$. Track recognition and reconstruction starts downstream. The PR forms and fits > 3 consecutive hit chains. Finally the chains are joined to form tracks. The existing MPS vertex finding and fitting program has been adapted to the TPC analysis. High efficiency track reconstruction $>95 \%$ has been attained in Monte Carlo's and actual MPS and beam tests which were monitored on-line.

The acceptance in the forward half of the nucleon-nucleon $\mathrm{cm}$ rapidity region (i.e. $y_{1 \mathrm{ab}}=1.75$ to 3.5 correspoonding to $\mathrm{y}_{\mathrm{cm}}=$ $0-1.75$ in the $c m$ system which extends from central to maximum rapidity). We get excellent $(<0.1$ unit) pseudorapidity and rapidity 


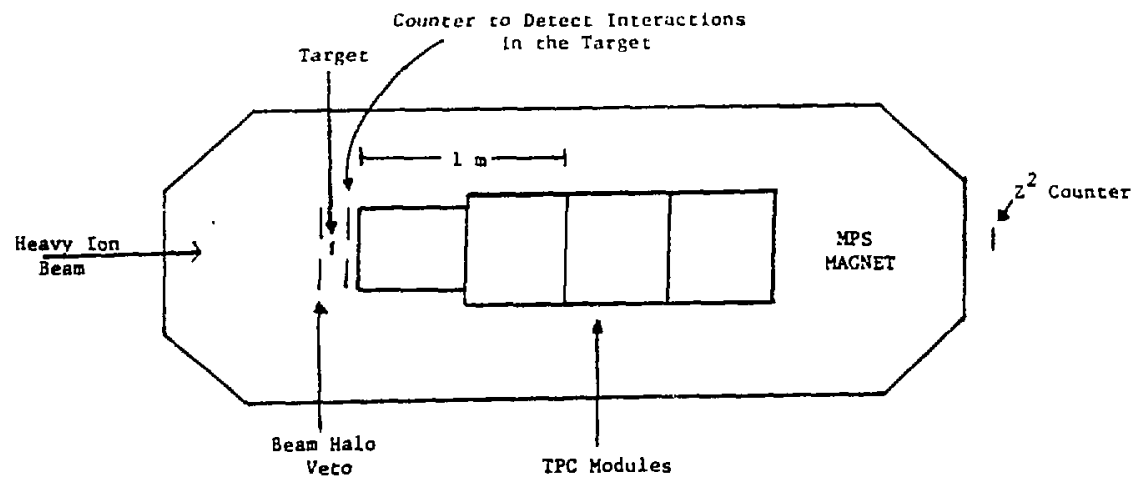

NOTE: Counters and target are no: to scale

Figure 2: AGS E-810 Setup

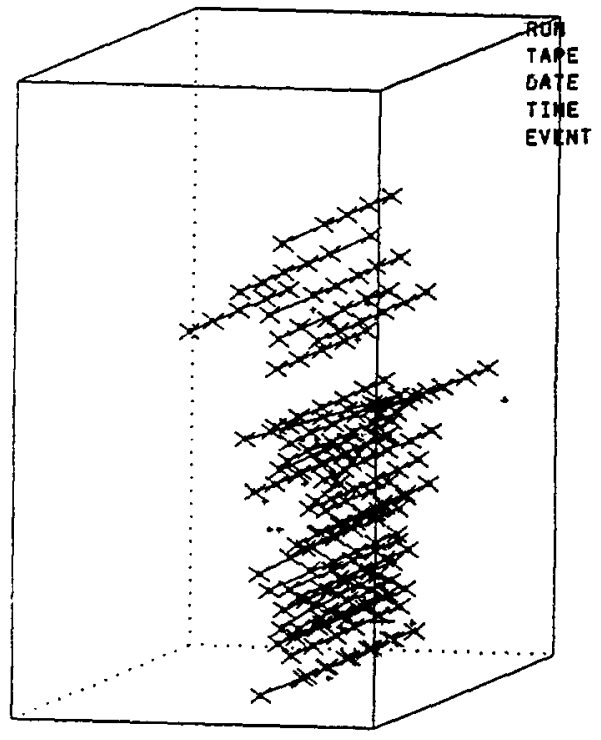

Fig. 3a: High Rate $18 \mathrm{GeV} / \mathrm{c}$ Protons

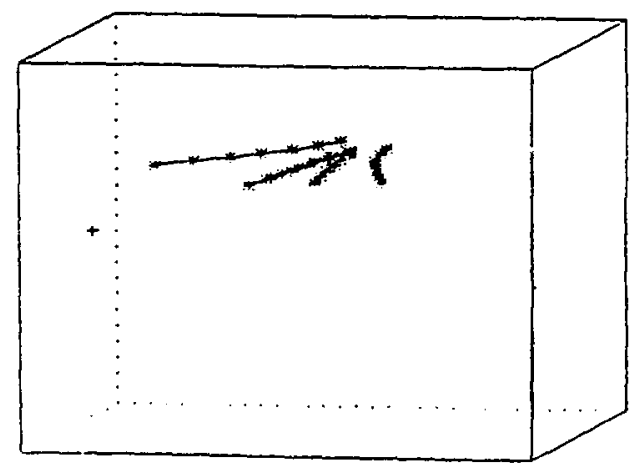

18 GEV/C Proton Beam 5 CM BRASS TARGET

( $20 \mathrm{cM}$ (JPSTREAM)
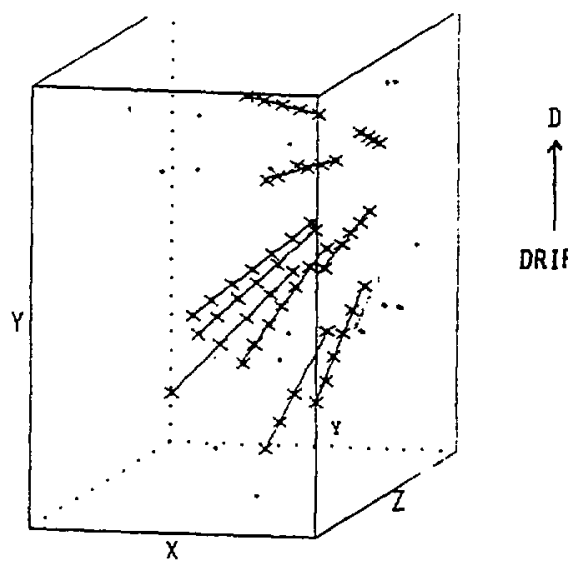

DRIFT

Fig. 3b: Interaction Detected

Fig. 3c: TPC in MPS Magnet 
resolution (when particles are identified). Fig. 4 shows that central cascade and plasma events have similar enough rápidity distributions that we cannot use those as a plasma signal.

Figure 5 shows that the multiplicity distributions for cascade and plasma events are similar except that plasma events are shifted toward lower multiplicity by about $20 \%$ because they have enhanced production of heavier objects such as $\bar{p} p$ pairs, $\bar{\lambda}$, and $K_{S}$, etc. Thus multiplicity cannot be used as a plasma detector. Comparisons between plasma and central cascade transverse and longitudina? momentum distribution of $\pi^{-}$show no essential difference. Thus they cannot be used as a plasma detection signal.

\section{PLASMA SIGNALS}

However we did find that $\bar{p} \bar{\Lambda}$ and $\bar{p} \bar{\Lambda}+$ Yees from the same event provide an excellent plasma signal which is detectable.

The $\vec{\Lambda}$ can be identified by cuts which ensure the $\bar{\Lambda}$ vertex or particles came from the interaction point in the target and that the $\bar{\Lambda}$ momentum points to the interaction point. $K_{S}$ and $\Lambda$ can be identified in a similar manner. The results of this type of kinematic identification obtained by our Monte Carlo simulation for plasma events and for central events shows that we can identify $\Lambda$ and $K_{s}$ about an order of magnitude above background. In plasma events this is also true for $\bar{\Lambda}$. About half the $\bar{p}$ are identified by the time-of-flight hodoscope referred to previously. This will further improve the signal-to-background ratio.

In Table I we compare various plasma singals (according to the model described) in events with plasma and events with no plasma per $10,000 \mathrm{~S}$-Au central events. Since we plan to gather $-50,000$ effectively central collision events. If $-5 \%$ of the central collisions produce plasma in an effectively central region covering a radius of $r \leq 1.5$ fermi, we should expect to observe the following: 1) 37 event $\bar{s}$ containing a $\bar{p} \bar{\Lambda}$ with a background of $=10 ; 2) 12$ events containing $\bar{p} \bar{\Lambda}$ and $1 \vee$ with background -1 ; 3) 2.5 events

\section{TABLE I - PLASMA SIGNALS}

Detected Event Rates and Background Rates per 10,000 $S-A u$ Central Events

Reaction

Signal

Events

Selector

\begin{tabular}{ccr} 
Required & With Plasma & No Plasma \\
\cline { 3 - 3 }$\overline{\bar{p}} \bar{\Lambda}$ & 150 & 1.0 \\
$\bar{p} \bar{\Lambda}$ and 1V & 50 & 0.1 \\
$\bar{p} \bar{\Lambda}$ and 2V & 10 & 0.01 \\
$\bar{p} \bar{\Lambda}$ and $3 V$ & 2 & 0.001
\end{tabular}

The background due to fake $\bar{\Lambda}$ is expected to be comparable with the events with no plasma. 


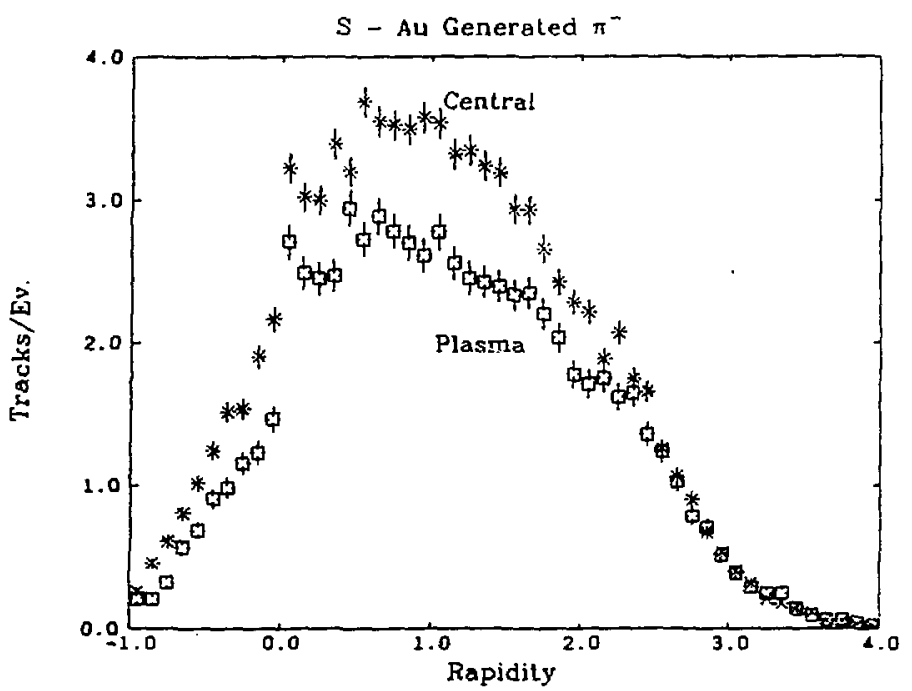

Figure 4: Rapidity Distribution

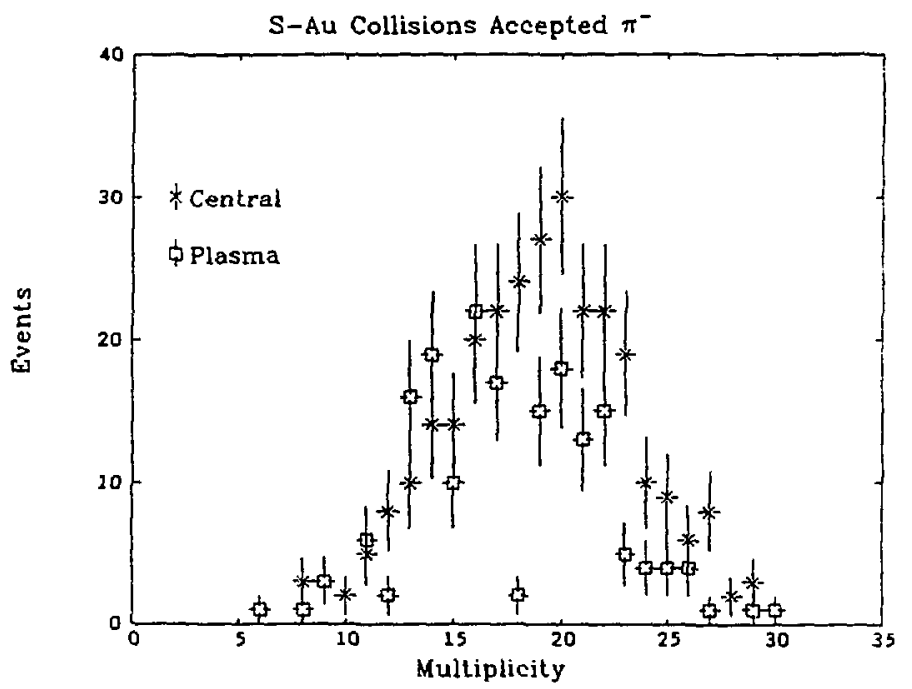

Figure 5: Multiplicity 
containing $\bar{p} \bar{\Lambda}$ and 2 Vees with a background of $\sim 0.1$; 4$)=1 / 2$ event $\bar{p} \bar{\Lambda}$ and $3 V$ with a background -0.01 .

We plan to run with several targets including $p, 0$, and Si beams. We obviously can increase our running time especially to detect reactions (3) and (4). We have also from our Monte Carlo's shown that we detect Hanbury-Brown twiss effects. We have used a particular plasma model to show the power of our technique. Nature may well reveal a different scenario. However we have a technique which is very well suited to detect any hadronic anomolies ad in fact we plan to use similar techniques for RHIC detectors."

It should be noted that while some of the TPC module tests previously reported were being made in the heavy ion beam some preliminary data on $A$ production was gathered in $\mathrm{Si}+\mathrm{Al}$ collisions using MPS II with its drift chambers. Figure 6 shows the clear $\Lambda$ peak.

Since the conference we have just now in progress tests of 15 $G e V / C \times A$ ion beams incident on Al etc. targets. The preliminary. results for TPC with MPS magnetic field detection of the events are satisfactory.

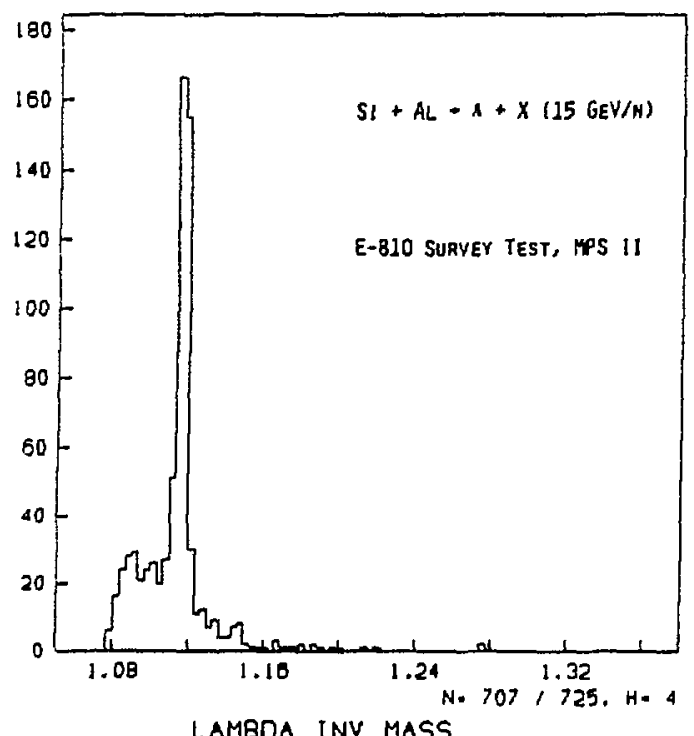

Figure 6:

A Peak with Drift Chambers

SUMPARY

1) We have improved HIJET in the following respects: a) We have modified ISAJET to conserve $B, S, E$, $P$, etc; $b$ ) Included cascading of $\mathrm{N}-\mathrm{N}, \pi-\mathrm{N}$ and $\mathrm{K}-\mathrm{N}$; c) Interfaced, the fragmentation program FAIHI; d) Used our model for plasma formation and treated plasma breakup according to Koch, Muller and Rafelski. 
2. a) We have found with these models that at AGS energies the best plasma signals that are clearly detectable are $\bar{p} \bar{\Lambda}$ and $\bar{p} \bar{\Lambda}$ plus Vees. We can detect these effects at a level of < $5 \%$ plasina events with $r \leq 1.5$ fermi for effectively central collisions using the TPC spectrometer system of AGS E-810. Hanbury-Brown Twiss effects can also be detected. $>90 \%$.

3. Tests show the TPC works well with reconstruction efficiency

4. AGS E-810 data run is planned for fall of 88 .

5. A Phase II of this program has been planned to fill a much larger part of the MPS magnet with a TPC system to increase the acceptance.

6. Our plasma and cascade event generators are being used for RHIC calculations.

7. We have proposed a large TPC spectrometer for RHIC. ${ }^{4}$

\section{REFERENCES}

1. Abbot et al., Phys. Lett. B 197,285 (1987).

2. Koch, Maller and Rafelski, Phys. Reports, February 1986.

3. Lindenbaum et a1., Nuc 1. Phys. A461, 431 (1987).

4. Lindenbaum, S.J. An Approximately $4 \pi$ Tracking Spectrometer for RHIC, Proc. of Second Workshop on Experiments and Detectors for RHIC, [BL, Berkeley, California, May 25-29, 1987). 